\title{
Origin of the mixed alkali effect in silicate glass
}

\author{
Yohei Onodera ${ }^{1,2}$, Yasuyuki Takimoto ${ }^{3}$, Hiroyuki Hijiya ${ }^{4}$, Taketoshi Taniguchi ${ }^{3}$, Shingo Urata ${ }^{3}$, Seiji Inaba ${ }^{4}$, Sanae Fujita ${ }^{3}$, \\ Ippei Obayashi, ${ }^{5,6}$, Yasuaki Hiraoka, ${ }^{7,6,1}$ and Shinji Kohara $8,1,9,10$
}

\begin{abstract}
Silicate glasses have evolved from basic structural materials to enabling materials for advanced applications. In this article, we unravel the origin of the mixed alkali effect for alkali silicate $22.7 \mathrm{R}_{2} \mathrm{O}-77.3 \mathrm{SiO}_{2}$ glasses $(\mathrm{R}=\mathrm{Na}$ and/or $\mathrm{K})$ by identifying the variation in the alkali ion location around the non-bridging oxygen atoms. To do so, we constructed a state-of-the art structural model, which reproduces both diffraction and NMR data with a particular focus on the behavior of the alkali ions. A novel topological analysis using persistent homology found that sodium-potassium silicate glass shows a significant reduction in large cavities as a result of the mixed alkali effect. Furthermore, a highly correlated pair arrangement between sodium and potassium ions around non-bridging oxygen atoms was identified. The potassium ions can be trapped in $\mathrm{K}-\mathrm{O}$ polyhedra due to the increased bridging oxygen coordination; therefore, the correlated pair arrangement is likely the intrinsic origin of the mixed alkali effect.
\end{abstract}

\section{Introduction}

Silicate glasses are one of the oldest materials in history and are commonly used in the modern glass industry. They have varying physicochemical properties, such as melting point, glass transition temperature, viscoelasticity, thermal expansion and chemical durability with the addition of the other network forming cations, such as boron and aluminum ions, and the network modifiers, namely, alkali metal or alkali earth metal ions. They are typically manufactured as window glasses for buildings and automobiles and display glasses for TV panels and mobile phones.

It is well known that a short-range structural unit in silica $\left(\mathrm{SiO}_{2}\right)$ and silicate glasses is a $\mathrm{SiO}_{4}$ tetrahedron. Unlike the structure of crystalline materials such as quartz or cristobalite, silica glass does not have long-range periodicity, and the structure can be described as a network of cornersharing and randomly connected $\mathrm{SiO}_{4}$ tetrahedra via bridging oxygen on the basis of Zachariasen's random

\footnotetext{
Correspondence: Shinji Kohara (KOHARA.Shinji@nims.go.jp)

${ }^{1}$ Center for Materials Research by Information Integration (CMI2), Research and Services Division of Materials Data and Integrated System (MaDIS) National Institute for Materials Science (NIMS), Ibaraki 305-0047, Japan

${ }^{2}$ Institute for Integrated Radiation and Nuclear Science, Kyoto University, 2-1010 Asashiro-nishi, Kumatori-cho, Sennan-gun, Osaka 590-0494, Japan Full list of author information is available at the end of the article.
}

network theory ${ }^{1}$. On the other hand, the formation of an intermediate-range order in disordered materials, including silica glass, is indicated by the first sharp diffraction peak (FSDP) in neutron diffraction (ND) and X-ray diffraction (XRD) patterns ${ }^{2-4}$. The FSDP of silica glass has been extensively investigated by ND/XRD and molecular dynamics (MD) simulations ${ }^{5-7}$, and it has been found that the three-dimensional network possesses a periodicity $(=$ $\left.2 \pi / Q_{\mathrm{FSDP}}\right)$ of $\sim 4 \AA$ and correlation length $\left(=2 \pi / \Delta Q_{\mathrm{FSDP}}\right)$ of $\sim 10 \AA$, which is associated with the formation of large cavities.

It is possible to infer that the introduction of alkali ions into $\mathrm{SiO}_{2}$ glass causes partial cleavage of the tetrahedron network, and thus, the numbers of non-bridging oxygen (NBO) and silicon with $\mathrm{Q}^{n}(n<4)$ (where $n$ is the number of bridging oxygen atoms in $\mathrm{SiO}_{4}$ tetrahedron $)^{8}$ increase by breaking the silicon-oxygen bonds. Consequently, the cavity volume decreases since the alkali ions occupy the cavity site. Contrary to a simple structural change, interpretation of the FSDP in binary alkali silicate glasses is rather complicated. This is because the FSDP mainly consists of at least six partial correlations in binary alkali silicate glasses, and hence, the aid of computer simulation is helpful. Previous MD simulation studies ${ }^{9,10}$ have suggested that the ring structure formed by $\mathrm{SiO}_{4}$ tetrahedra is 
affected by alkali ions, resulting in broadening of the ring size distribution. Although theoretical simulations claim that the FSDP position and width are modified by alkali ions ${ }^{10}$, they fail to reproduce the FSDP shape experimentally observed by neutron diffraction ${ }^{11,12}$. In particular, in the case of potassium silicate glass, the FSDP in the neutron diffraction data is not a simple peak ${ }^{11}$, and it is not easily reproduced by MD simulations.

The structure of multicomponent alkali silicate glasses under solid and melted conditions has also been extensively studied using various experimental techniques, such as $\mathrm{NMR}^{8,13,14}$ and Raman spectroscopy ${ }^{15,16}$, quasielastic neutron scattering ${ }^{17,18}$, and positron annihilation spectroscopy ${ }^{19}$. However, it is still difficult to fully understand the intermediate-range order in alkali silicate glasses solely using these experimental methods. To reveal the effect of alkali ions on the silicate network in the intermediate range, it is necessary to visualize a reliable atomistic model while maintaining consistency between the theoretical atomistic model and the experimental diffraction data.

In this work, we therefore try to unravel the atomistic structure of three kinds of $22.7 \mathrm{R}_{2} \mathrm{O}-77.3 \mathrm{SiO}_{2}$ glasses $(\mathrm{R}=$ $\mathrm{Na}$ and/or K) using reverse Monte Carlo (RMC) modeling in conjunction with ND and high-energy XRD (HEXRD). To reveal the mixed alkali effect, single $\mathrm{Na}$ (Na100 glass), single $\mathrm{K}$ (K100 glass) and an equal mixture of $\mathrm{Na}$ and $\mathrm{K}$ ions (Na50K50 glass) were investigated. Since both $\mathrm{Na}$ and $\mathrm{K}$ ions are abundant in the Earth's crust and essential for modifying the physical and chemical properties of glass, it is worth investigating the intrinsic origin of the mixed alkali effect, which might arise from the difference in ionic radii. Indeed, it is well known that alkali mixing causes several unusual phenomena; that is, when an alkali ion is gradually replaced by another one, some physical properties vary in an extremely nonlinear trend. For instance, a composition containing equally mixed alkali ions has a pronounced maximum electrical resistivity and a substantially lower viscosity as a concave function of the alkali content, although the variation in the molar volume is almost linear with the alkali content ${ }^{20}$. These facts suggest that ionic migration is inhibited by the alkali mixing associated with the formation of NBO atoms in a modified network model of alkali silicate glasses proposed by Greaves ${ }^{21}$. A large number of studies investigating this phenomenon have been reported ${ }^{22-24}$, and schematic models involving NBO atoms for binary alkali oxide glasses have been proposed ${ }^{21,25,26}$. The details of the previous studies are reviewed by Habasaki et al. ${ }^{27}$ Nevertheless, the origin of the mixed alkali effect remains unclear because the previous simulation studies did not visualize the alkali ion coordination around the NBO atoms in the structural model to be consistent with the multiple experimental dataset, e.g., diffraction and NMR. Therefore, it is worthwhile to revisit the glass structure with a special focus on the alkali ion coordination around the NBO atoms towards reconciling the mixed alkali effect.

In this article, we measured X-ray and neutronweighted structural factors of the aforementioned three alkali silicate glasses, whose dielectric constants show a clear mixed alkali effect (Fig. S1), using state-of-the art techniques. The diffraction data were utilized to reconstruct atomistic views of the glasses using a combination of RMC and MD simulations (RMC-MD) in conjunction with the NMR data ${ }^{8}$. For the RMC-MD simulation, proper constraints for the coordination numbers of silicon, bond angle distribution of $\mathrm{O}-\mathrm{Si}-\mathrm{O}$, partial pair-distribution functions within the first coordination shell, and $\mathrm{Q}^{n}$ distributions were adopted to avoid physically unreasonable configurations. The optimized atomistic models were investigated by a sophisticated topological analysis based on persistent homology to highlight the modification of the glass structure by alkali mixing, in addition to the ordinal ring size and cavity size distribution analyses. We especially focused on the behavior of the alkali ions around the NBO atoms, as well as that of the cavities in the glasses to tackle the intrinsic origin of the mixed alkali effect.

\section{Materials and methods \\ Materials}

Alkali silicate glasses were prepared from mixtures of reagent grade $\mathrm{SiO}_{2}, \mathrm{Na}_{2} \mathrm{CO}_{3}$, and $\mathrm{K}_{2} \mathrm{CO}_{3}$ powders. The mixtures were melted at $1923 \mathrm{~K}$ in air. Each melt was quenched and annealed at the glass transition temperature and then cooled slowly by $1 \mathrm{~K} / \mathrm{min}$. The nominal and analyzed compositions of the glasses are listed in Table S1. The alkali ion contents were determined by a Z-2310 atomic absorption spectrophotometer (Hitachi High-tech Science Corp.) in aqueous solutions of the decomposed glasses, and the densities were measured with the Archimedes method.

\section{Diffraction measurements}

The ND experiments were conducted on a high intensity total diffractometer, NOVA ${ }^{28}$, installed at BL21 of the Materials and Life Science Experimental Facility at the JPARC spallation neutron source. Crushed glass samples were transferred into vanadium-nickel null alloy cells $6 \mathrm{~mm}$ in diameter. The wavelength range of the incident neutron beam was $0.12 \AA<\lambda<8.3 \AA$.

The observed scattering intensities for the samples were corrected for instrumental background, absorption of the samples and cell, and multiple and incoherent scattering and then normalized by the incident beam profile.

The HEXRD experiments were performed on a two-axis diffractometer, which is dedicated for disordered materials, at the BL04B2 beamline of the SPring- 8 synchrotron 
radiation facility ${ }^{29}$. Crushed glass samples were transferred into $\mathrm{SiO}_{2}$ glass capillaries. The wavelength of the incident X-ray was $0.2011 \AA$. The raw data were corrected for polarization, absorption and background, and the contribution of Compton scattering was subtracted using standard data analysis software ${ }^{29}$.

The fully corrected data were normalized to give a Faber-Ziman ${ }^{30}$ total structure factor $S(Q)$. Total correlation functions, $T(r)$, were obtained by a Fourier transform with a Lorch function ${ }^{31}$.

\section{Modeling}

The RMC modeling of the Na100, Na50K50, and K100 glasses was performed using the $\mathrm{RMC}++\operatorname{code}^{32}$. The atomic number densities assumed were $0.0728 \AA^{-3}$, $0.0687 \AA^{-3}$, and $0.0642 \AA^{-3}$ for the Na100, Na50K50, and K100 glasses, respectively. The starting configurations, which contain 3300 particles for the three glasses, were created using hard-sphere Monte Carlo (HSMC) simulations with constraints to avoid physically unrealistic structures. The $r$-spacing for the calculations of partial pairdistribution functions was set to $0.05 \AA$. Two kinds of constraints were applied: the closest atom-atom approach and the $\mathrm{Si}-\mathrm{O}$ connectivity. The first one can avoid unreasonable spikes in the partial pair-distribution functions. The second forces silicon atoms to coordinate to four oxygen atoms within a cutoff distance of $1.90 \AA$. In addition, the fixed neighbor constraints ${ }^{33}$ were applied for the $\mathrm{Si}-\mathrm{O}$ correlation at 1.45-1.90 $\AA$ to reproduce the $Q^{0}: Q^{1}: Q^{2}: Q^{3}: Q^{4}$ ratio obtained by the NMR study ${ }^{8}$. According to the NMR measurements, the $\mathrm{Q}^{0}: \mathrm{Q}^{1}: \mathrm{Q}^{2}: \mathrm{Q}^{3}: \mathrm{Q}^{4}$ ratio is $0: 0: 1.5: 55.8: 42.7$, $0: 0: 1.2: 56.5: 42.4$, and 0:0:0.8:57.2:42.0 for the Na100, Na50K50 and K100 glasses, respectively. The ratios obtained by the RMC-MD modeling were 0:0:2.2:54.4:43.4, $0: 0: 2.5: 54.7: 42.8$, and 0:0:3.2:53.2:43.6 for the Na100, $\mathrm{Na} 50 \mathrm{~K} 50$ and K100 glasses, respectively. After the HSMC simulations, the RMC simulations were conducted to reproduce the X-ray $S(Q)$ and neutron $S(Q)$ data. Following the RMC simulations, the atomic configurations were optimized by the MD simulations.

The MD simulations were performed using the LAMMPS package ${ }^{34}$ with the Morse-type interatomic potential developed by Pedone et al..$^{35}$. For the alkali silicate glasses, the structures obtained by the RMC simulations were optimized by minimizing the energy using the conjugate gradient method. During the optimization, the volume was kept constant. In the case of silica glass, a random configuration composed of 3000 atoms was prepared with respect to the experimental density, and then, an equilibrium configuration was constructed using the melt quenching method. That is, following the melting simulation at $3500 \mathrm{~K}$ for $500 \mathrm{ps}$, the model was cooled down to $300 \mathrm{~K}$ with a quenching rate of $1 \mathrm{~K} / \mathrm{ps}$. Eventually, the system was equilibrated at $300 \mathrm{~K}$ for 300 ps. The equation of motion was integrated with a $1 \mathrm{fs}$ time step with the canonical ensemble (NVT). A Nosé-Hoover thermostat ${ }^{36}$ was employed to control temperature.

After the MD simulations, all configurations were refined by additional RMC simulations while constraining the coordination number of silicon, the bond angle distribution for $\mathrm{O}-\mathrm{Si}-\mathrm{O}$, and the partial pairdistribution functions within the first coordination shell to avoid formation of an unfavorable disordered structure.

\section{Topological analyses}

The primitive $(\mathrm{Si}-\mathrm{O})_{n}$ ring size distributions for the alkali silicate glass were calculated using the R.I.N.G.S. $\operatorname{code}^{37}$. The surface cavity analysis was performed employing the pyMolDyn code ${ }^{38}$ with a cut off distance $r_{\mathrm{c}}$ $=2.5 \AA$. The persistent homology analyses were performed using the HomCloud package ${ }^{39}$. Given a set of points in space, persistent homology captures the topological multiscale structures, and those identified are compactly expressed in a persistence diagram. The construction of the persistence diagram follows the process schematically described in Fig. 1a. We first replace each point with a sphere and increase the radius from zero to a sufficiently large value, which corresponds to the changing resolution of our input $x, y$, and $z$ coordinates of atoms. Then, we record the pair of radii $(b, d)$ at which a ring in a specific location appears (birth) and disappears (death). The persistence diagram is a histogram of the birth-death plane counting of rings at the coordinate $(b, d)$. The diagram enables one not only to count the number of rings but also to characterize those shapes at multiscales. Typical examples of birth-death pairs for typical regular structures are depicted in Fig. 1b-d. For regular hexagonal points whose distance between points is $a$, the ring appears at radius $a / 2$ and disappears at radius $a$, as shown in Fig. 1b. For a regular triangular configuration, the ring appears at $a / 2$ and disappears at $\sqrt{\frac{1}{3}} a \approx 0.577 a$, as shown in Fig. 1c, and the onedimensional persistence diagram for regular hexagonal/ triangular points is shown in Fig. 1d. Further details are described in ref. ${ }^{40}$. In this article, persistence diagrams are used to investigate rings and polyhedral formations in atomic configurations. We also note that the detected rings are recorded during the computation of the diagrams, and hence, we can explicitly identify their geometric shapes.

\section{Results and discussion \\ The mixed alkali effect}

Figure S1 shows the dielectric constants, $\varepsilon_{r}$, for the alkali silicate glasses measured at different frequencies. It is well 


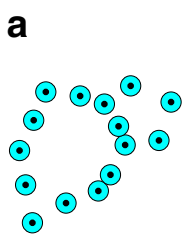

No ring

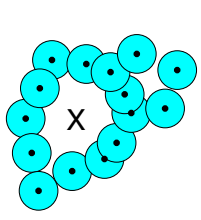

A ring $X$ appears $r=b_{1}$

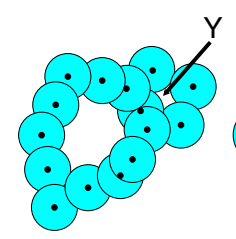

Another ring $Y$ appears $r=b_{2}$

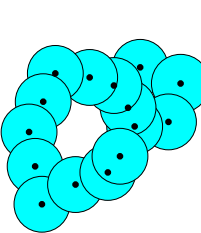

$\mathrm{Y}$ disappears

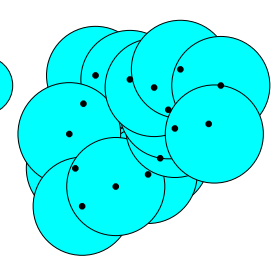

$X$ disappears

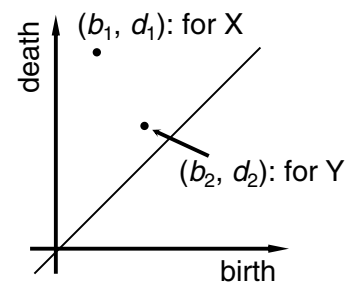

b

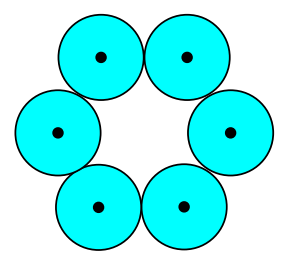

birth $(r=a / 2)$

C

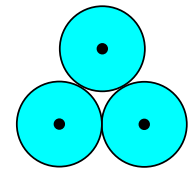

birth $(r=a / 2)$

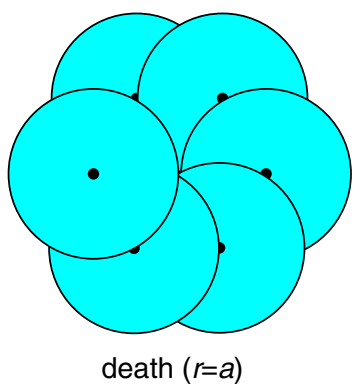

d

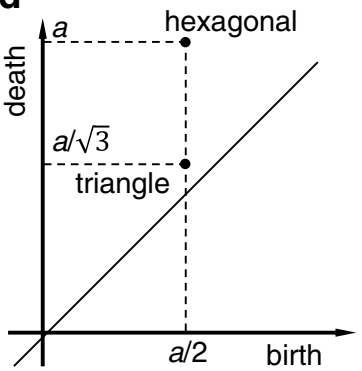

Fig. 1 Persistent homology and persistence diagram. a The increasing sequence of spheres for input data (left). The persistence diagram (right) is obtained as a histogram counting the number of rings on the birth-death plane. $\mathbf{b}, \mathbf{c}$ The appearance and disappearance of a ring for a regular hexagon/triangle. $\mathbf{d}$ The pairs of birth and death radii for a hexagon and triangle in the one-dimensional persistence diagram.

known that mixed alkali glasses show a pronounced reduction in dc conductivity in comparison with single alkali glasses ${ }^{20,41}$. Indeed, the $\varepsilon_{r}$ shown in Fig. S1 exhibits a nonlinear trend as a function of the $\mathrm{K}_{2} \mathrm{O}$ amount and has a minimum value in the Na50K50 glass at all frequencies. It is also observed that the mixed alkali effect diminishes with increasing frequency. This behavior is in agreement with the previous study on the $33.3\left(\mathrm{Na}_{2} \mathrm{O}+\mathrm{Li}_{2} \mathrm{O}\right)$ $66.7 \mathrm{SiO}_{2}$ system $^{41}$. Thus, the mixed alkali effect is clearly observed in the electrical properties of the alkali silicate glasses.

\section{Structure factors and real-space functions}

Figure 2 compares the neutron and X-ray structure factors, $S^{\mathrm{N}, \mathrm{X}}(Q)$, between the alkali silicate glasses and $\mathrm{SiO}_{2}$ glass. A sharp FSDP is observed at $Q \sim 1.5 \AA^{-1}$ in the $S^{\mathrm{N}, \mathrm{X}}(Q)$ for $\mathrm{SiO}_{2}$ glass, whereas the FSDP becomes broader when alkali ions are added. The FSDP also appears in the $S^{\mathrm{N}}(Q)$ for the alkali silicate glasses, but the shape of the peak is very complicated. Indeed, an extra peak is observed at $Q \sim 0.8 \AA^{-1}$ in the case of the K100 glass. On the other hand, the FSDP is not visible in the $S^{\mathrm{X}}(Q)$ for both the Na50K50 and K100 glasses due to the large weighting factors of potassium for $\mathrm{X}$-rays. The second principle peak (PP) is found only in the $S^{\mathrm{N}}(Q)$ because the PP reflects the oxygen-oxygen correlation, which is easily detected by neutrons. It is worth noting that an average of the diffraction profiles of the Na100 and K100 glasses (black broken curve) is almost identical to the experimental data for the Na50K50 glass (green curve) for both $S^{\mathrm{N}}(Q)$ and $S^{\mathrm{X}}(Q)$, implying that the mixed alkali effect cannot be detected easily in the diffraction data. Readers can find the enlarged figures in Fig. S2, which show only subtle differences.

The neutron and X-ray total correlation functions, $T^{\mathrm{N}, \mathrm{X}}(r)$, for the alkali silicate glass are shown in Fig. 3. The $\mathrm{Si}-\mathrm{O}$ correlation peak is located at $1.62 \AA$ with a $\mathrm{Si}-\mathrm{O}$ coordination number of $4.0 \pm 0.1$ in all glasses, and the $\mathrm{Na}-\mathrm{O}$ correlation exhibits a small peak at approximately $2.3 \AA$ in the cases of the Na100 and Na50K50 glasses. The $\mathrm{O}-\mathrm{O}$ correlation peak and the $\mathrm{Si}-\mathrm{Si}$ correlation peak are found at $2.63 \AA$ and $3.08 \AA$, respectively, in $\mathrm{SiO}_{2}$ glass, but the peak overlaps with the K-O correlation peak for the Na50K50 and K100 glasses. It is expected from the $\mathrm{Si}-\mathrm{Si}$ correlation peak that a more complicated structure should be formed in the alkali silicate glasses. However, it is not easy to extract more detailed information beyond the short range according 

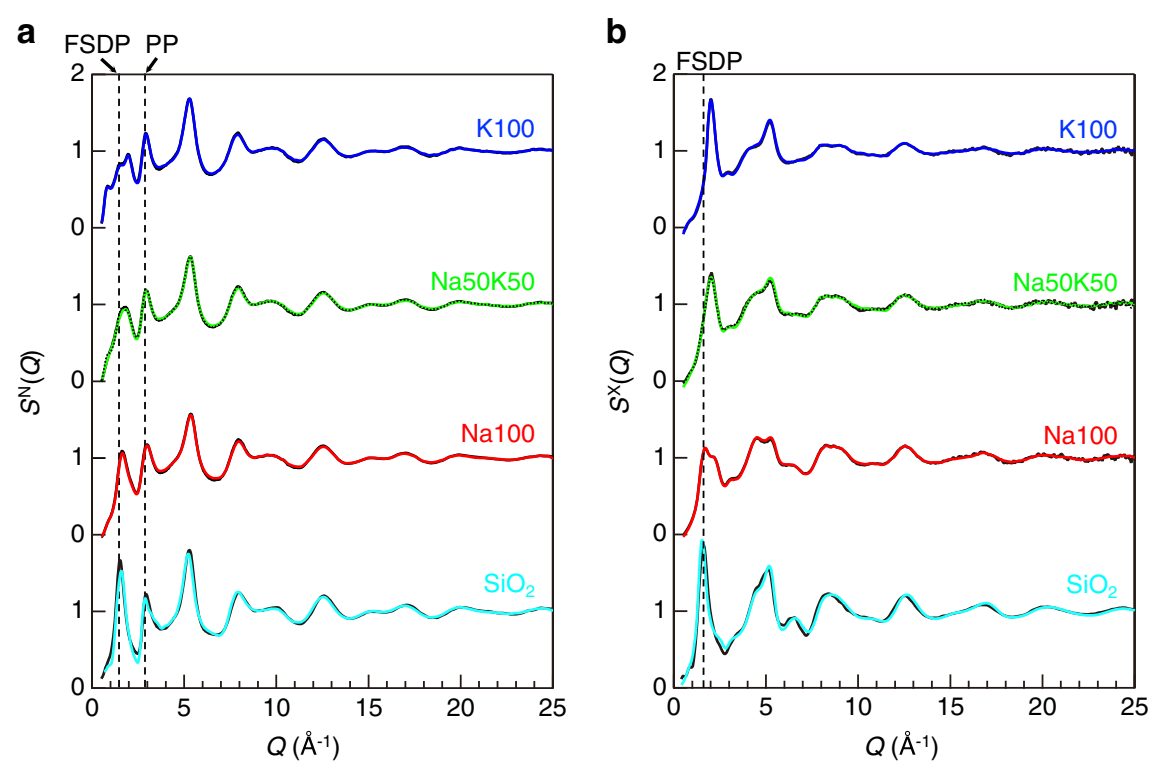

Fig. 2 Comparison between ND/HEXRD data and the RMC-MD model for the alkali silicate glasses in $Q$ space. a Neutron total structure factor $S^{N}(Q)$ and $\mathbf{b} X$-ray total structure factor $S^{X}(Q)$. Black solid curve, experimental data; colored curve, RMC-MD model. Averaged experimental $S^{N, X}(Q)$ for the Na50K50 glass calculated using the Na100 glass and K100 glass data are plotted as a dashed black curve. The RMC-MD and experimental data are practically indistinguishable. The $S^{N, X}(Q)$ for the Na50K50 glass is roughly identical to the average of those for the Na100 and K100 glasses. It is worth mentioning that both the FSDP and PP are visible in the neutron structure factors, $S(Q)$, while X-ray $S(Q)$ exhibits only the FSDP, because PP is largely taken by the oxygen-related correlation, which can be easily detected by neutrons.

a

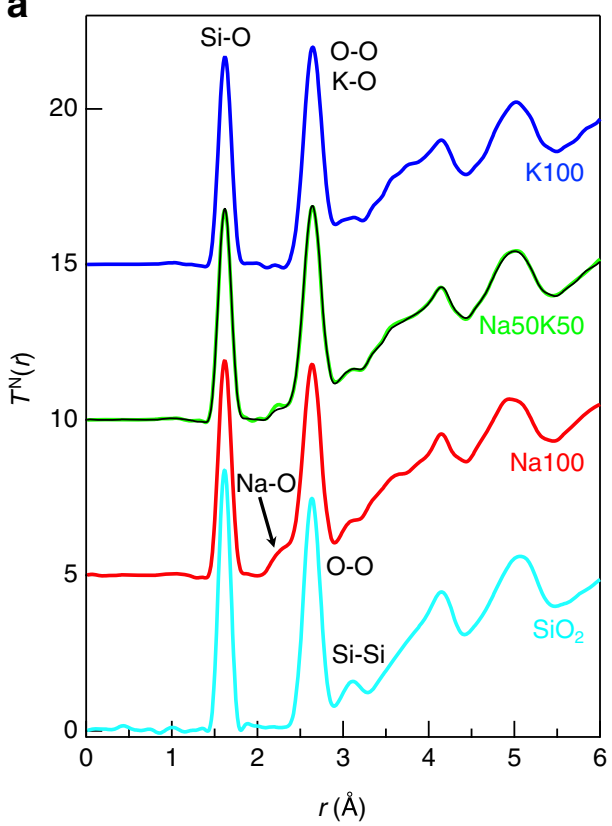

b

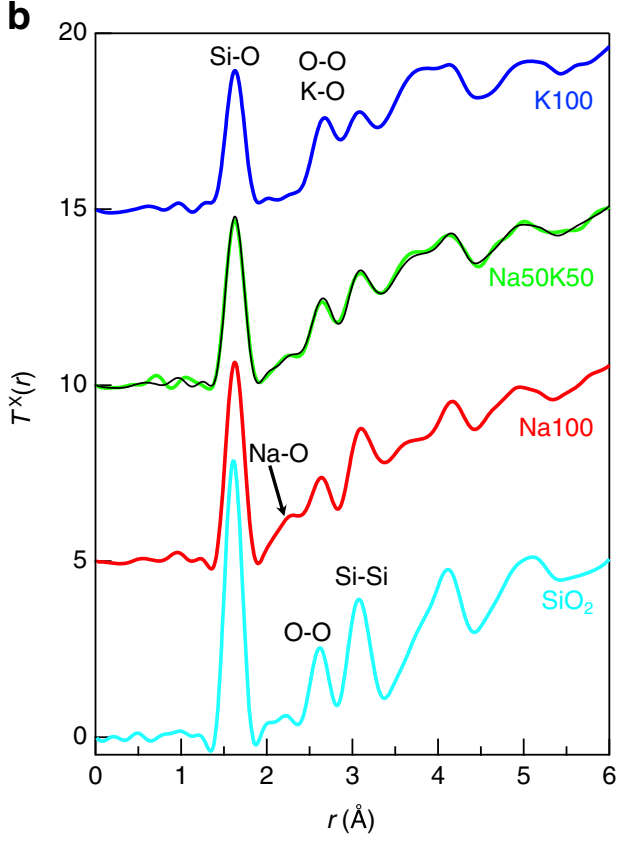

Fig. 3 Comparison between ND/HEXRD data and the RMC-MD model for the alkali silicate glasses in real space. a Neutron total correlation function $T^{N}(r)$ and $\mathbf{b}$ X-ray total correlation function $T^{\mathrm{X}}(r)$. Averaged experimental $T^{\mathrm{N}, \mathrm{X}}(r)$ for the Na50K50 glass calculated using the Na100 and K100 glass data are plotted as a black curve. $T^{\mathrm{N}, \mathrm{X}}(r)$ for the Na50K50 glass is approximately identical to the average of that for the Na100 and K100 glasses. The $Q_{\max }$ values in a Fourier transformation for ND and HEXRD data are $40 \AA^{-1}$ and $25 \AA^{-1}$, respectively. The contrast between neutron and X-ray data is excellent because neutrons are sensitive to oxygen-oxygen correlation, while the cation-related correlation can be easily detected by $\mathrm{X}$-rays. 
to real space analysis, demonstrating the limitation of pairwise real-space correlations, although elementspecific experimental real-space functions can extract long-range information ${ }^{42}$.

\section{Analysis of partial structure}

To obtain clear insight into the intermediate-range structure beyond the short range, we performed dedicated data-driven structure modeling with a combination of RMC and classical MD simulations. First, the atomic configurations, which reproduce the $\mathrm{Q}^{n}$ distributions measured by NMR spectroscopy ${ }^{8}$, for the alkali silicate glasses were constructed by the RMC modeling technique. After that, the obtained structures were optimized using the classical MD simulation and minimizing the energy and then eventually subsequent RMC to refine the configurations. The final results of the RMC-MD modeling are plotted in Fig. 2 as solid colored curves. Good agreement between the RMC-MD model and the experimental data is obtained.

Figure 4a-c compare the ND/HEXRD $S^{\mathrm{N}, \mathrm{X}}(Q)$ and the partial structure factors, $S_{i j}(Q)$ ( $i$ and $j$ are atoms), for the alkali silicate glasses, allowing us to find the contribution of any pairs of $S_{i j}(Q)$ to the overall FSDP and PP. The most striking features are the very prominent negative contributions of the alkali-alkali $(\mathrm{Na}-\mathrm{Na}, \mathrm{Na}-\mathrm{K}$, and $\mathrm{K}-\mathrm{K})$ partials to the FSDP. A similar negative contribution was reported in the result of the MD simulation for potassium disilicate glass ${ }^{10}$, but a negative trend is more apparent in our data. This is definitely a signature of the inhomogeneous distribution of the alkali ions ${ }^{14,17}$ and is strongly related to the variations in the partial pairdistribution functions, $g_{i j}(r)$, for the alkali-oxygen correlations (see Fig. S3). We would like to emphasize here that

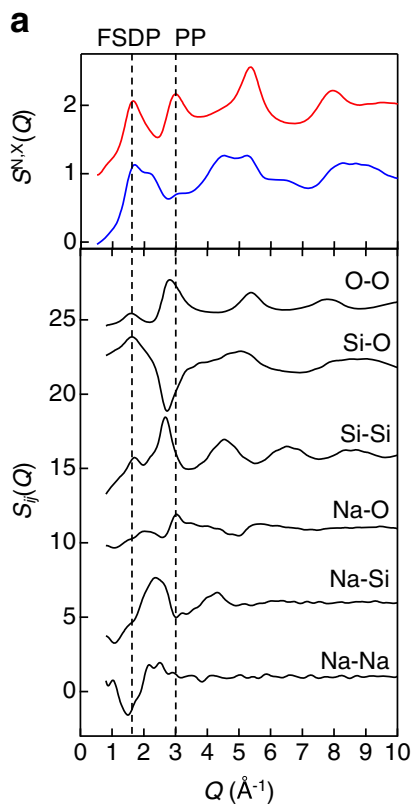

d

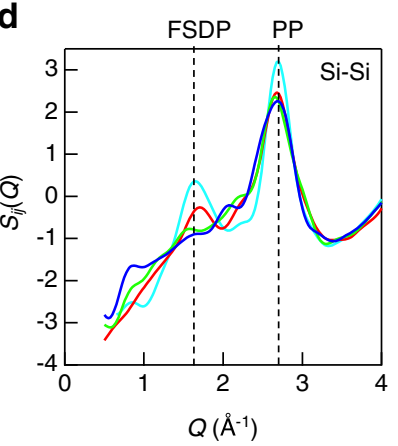

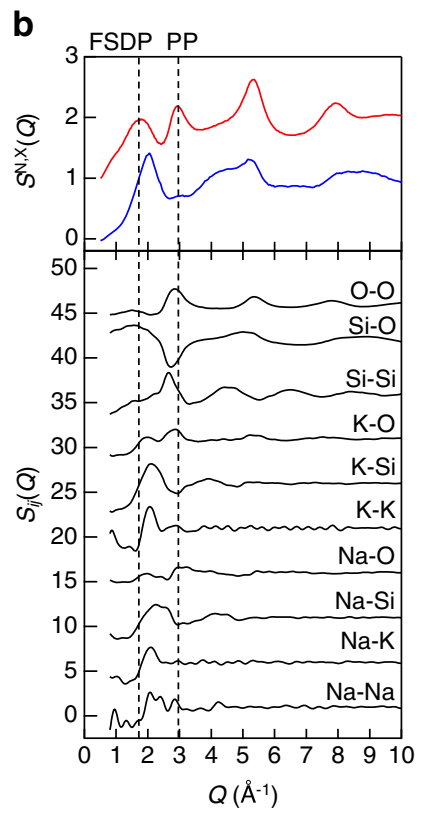

C
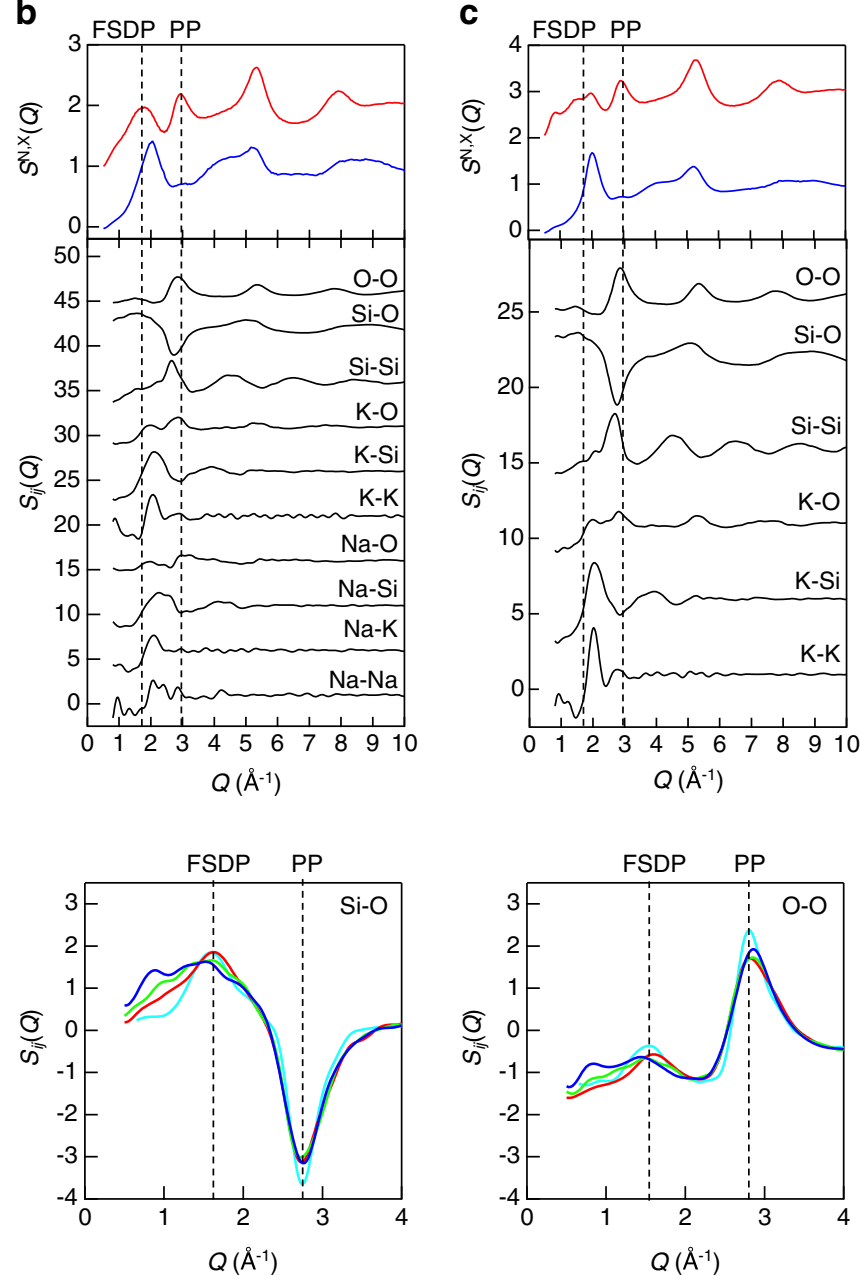

Fig. 4 Comparison between ND/HEXRD total and partial structure factors, $S_{i j}(Q)$ for the alkali silicate glasses. a Na100 glass, $\mathbf{b}$ Na50K50 glass, and c K100 glass. Red curve, ND data; blue curve, HEXRD data, d Partial structure factors, $S_{i j}(Q)$ for the network-forming components. Cyan curve; $\mathrm{SiO}_{2}$ glass, red curve; Na100 glass, green curve; Na50K50 glass, blue curve; K100 glass. The partial structure factors, $S_{i j}(Q)$ for $\mathrm{SiO}_{2}$ glass, exhibit a prominent positive contribution to the FSDP, while $S_{\text {sio }}(Q)$ has a negative contribution to the PP. 
the final RMC refinement of the experimental data enables us to visualize the intriguing variation in each correlation peak due to the finer structures in comparison with those obtained by the MD simulations (see Fig. S4). Comparisons of $S_{i j}(Q)$ for the network-forming components between $\mathrm{SiO}_{2}$ glass and the alkali silicate glasses are summarized in Fig. 4d. We can see prominent positive contributions to the FSDP from the $\mathrm{Si}-\mathrm{Si}, \mathrm{Si}-\mathrm{O}$, and O-O partial structure factors, $S_{i j}(Q)$, in the case of $\mathrm{SiO}_{2}$ glass. Intriguingly, the partial structure factors of $\mathrm{Si}-\mathrm{Si}, \mathrm{Si}-\mathrm{O}$, and $\mathrm{O}-\mathrm{O}$ for the $\mathrm{Na} 100$ glass have less of a contribution to the FSDP, but the contribution is still positive, suggesting that the sodium ion does not modify the Si-O network significantly due to its small size. We can hardly observe the positive contributions of the partial structure factors to the FSDP in both the Na50K50 and K100 glasses. Indeed, this is reasonable if we take account of the difference in the alkali ion size between sodium and potassium ions (approximately $0.4 \AA$ from the $T(r)$ data in Fig. 3 and the $g_{i j}(r)$ data in Fig. S3). Accordingly, it is possible to conclude that the extended-range order proposed by Price et al. ${ }^{43}$ can be formed only in the Na50K50 and K100 glasses.

\section{Analysis of the three-dimensional atomistic structure}

As discussed above, it is difficult to identify any detailed structural information on the basis of the pairwise correlation relating to the mixed alkali effect because the $S(Q)$ and $T(r)$ of Na50K50 glass are roughly identical to the average of those of Na100 and K100 glasses. Therefore, it is necessary to analyze the RMC-MD-generated atomistic configuration to extract structural information. Figure 5a (upper) depicts the primitive $(\mathrm{Si}-\mathrm{O})_{n}$ ring statistics. It is well known that $\mathrm{SiO}_{2}$ glass shows a broad ring size distribution ${ }^{44}$, although a six-atom ring, which is the only possible ring structure in the crystalline phase with comparable density (cristobalite), is dominant. This broad distribution is the result of topological disorder according to Cooper and Gupta ${ }^{45}$, and hence, this is a very important characteristic in glass. In the case of alkali silicate glasses, we can see a broader distribution due to the introduction of alkali ions breaking down the Si-O network, which is consistent with previous studies ${ }^{10,11}$. It is worth mentioning that the fractions of the larger rings $(n \geqq 13)$ increase with the addition of $\mathrm{K}_{2} \mathrm{O}$ (see Table 1), suggesting that $\mathrm{K} 100$ glass is the most topologically disordered. The cavity surfaces are visualized in Fig. 5b (middle). Obviously, $\mathrm{SiO}_{2}$ glass, which yields the largest cavity volume ratio of $32.2 \%$, exhibits a string-like cavity. The cavity

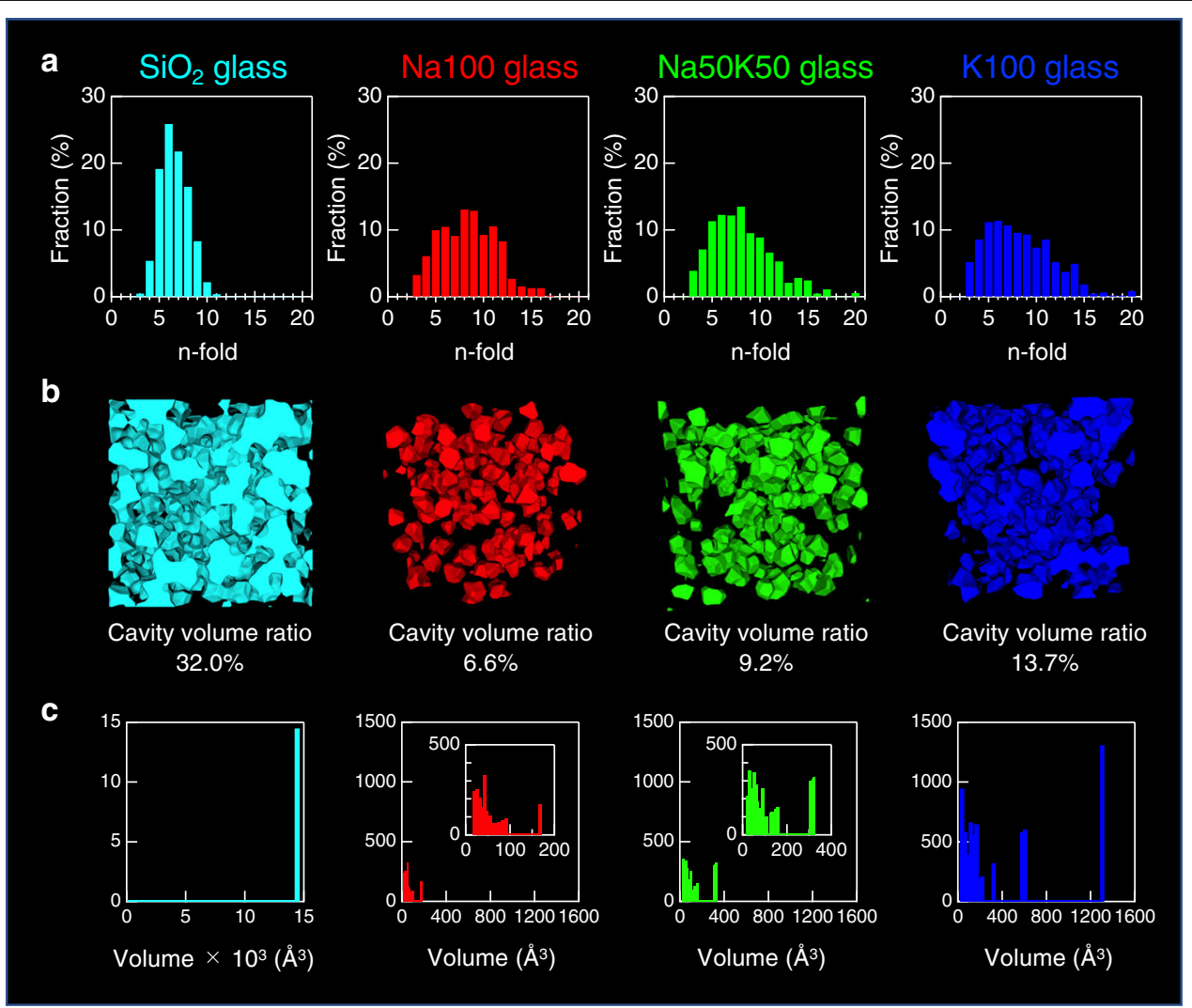

Fig. 5 Topological analyses for the alkali silicate glasses. a Primitive ring statistics, b visualization of surface cavities, c weighted surface cavity histograms. Cyan; $\mathrm{SiO}_{2}$ glass, red; Na100 glass, green; Na50K50 glass, blue; K100 glass. The insets highlight the distributions of small cavities. 
Table 1 Typical structural properties of the alkali silicate glasses.

\begin{tabular}{lllll}
\hline Glass & $\mathbf{S i O}_{2}$ & Na100 & Na50K50 & K100 \\
\hline Density $\left({\left.\mathrm{g} \cdot \mathrm{cm}^{-3}\right)}\right.$ & 2.200 & 2.429 & 2.439 & 2.404 \\
Number density $\left(\AA^{-3}\right)$ & 0.06615 & 0.07280 & 0.06866 & 0.06416 \\
$N_{\text {Na-O }}$ & - & 4.2 & 4.1 & - \\
$N_{\text {Na-BO }}$ & - & 2.1 & 2.0 & - \\
$N_{\text {Na-NBO }}$ & - & 2.1 & 2.1 & - \\
$N_{\text {K-O }}$ & - & - & 5.2 & 4.4 \\
$N_{\text {K-BO }}$ & - & - & 2.9 & 2.3 \\
$N_{\text {K-NBO }}$ & - & - & 2.3 & 2.1 \\
Average (Si-O) $n$ ring size & 6.6 & 8.3 & 7.9 & 8.1 \\
Fraction of large rings & 0 & 5.6 & 7.5 & 11.0 \\
$(n \geqq 13)$ (\%) & & & & \\
Cavity volume ratio & 32.2 & 6.6 & 9.2 & 13.7 \\
Maximum cavity volume $\left(\AA^{3}\right)$ & 14206 & 166 & 323 & 1310 \\
\hline
\end{tabular}

$B O$ bridging oxygen, $N B O$ non-bridging oxygen, coordination numbers were calculated up to $3.0 \AA$ and $3.3 \AA$ for $\mathrm{Na}-\mathrm{O}$ and $\mathrm{K}-\mathrm{O}$, respectively

volume ratio in the alkali silicate glasses increases monotonically with the increase in the $\mathrm{K}_{2} \mathrm{O}$ amount and cannot provide any evidence for the mixed alkali effect in the intermediate-range structure as well as the NMR data ${ }^{8}$. To shed light on the mixed alkali effect, we evaluated the cavity volume distribution in Fig. 5c (bottom), and the maximum cavity sizes in Table 1 . The volume of the largest cavity of $\mathrm{SiO}_{2}$ glass is $14,206 \AA^{3}$, and the maximum cavity volumes for the Na100, Na50K50, and K100 glasses are 166, 323, and 1,310 $\AA^{3}$, respectively, representing the nonlinear variation of the structure with increasing $\mathrm{K}_{2} \mathrm{O}$ content. Indeed, Fig. 5c (bottom) demonstrates that the maximum cavity size of the Na50K50 glass is obviously smaller than the average value of the Na100 and K100 glasses. Assuming that the size difference between sodium and potassium ions is $0.4 \AA$, the smaller size cavities should prevent the migration of potassium ions in the Na50K50 glass, which explains why the dielectric constant of the Na50K50 glass is the lowest, as shown in Fig. S1.

\section{Persistent homology analysis}

To obtain more detailed information to reconcile the mixed alkali effect, we applied a novel topological approach, the persistent homology analysis ${ }^{40}$, to extract the topology. Fig. S5a-c show the alkali-centric persistence diagrams for the alkali silicate glasses. We can observe two prominent profiles along the diagonal for all the glasses. It is known that the profile with a very short lifetime represents three-body correlations, as shown in Fig. S5d-f, and distinguishable differences among the three glasses are not observed in the short lifetime distributions, indicating that the short-range structures should be analogous. On the other hand, the alkali-centric persistence diagrams of $\mathrm{Na} 50 \mathrm{~K} 50$ glass for $\mathrm{Na}$-centric, $\mathrm{Na} / \mathrm{K}$-centric, and $\mathrm{K}$-centric data shown in Fig. 6 exhibit a striking difference. Both the Na-centric (Fig. 6a) and K-centric data (Fig. 6c) do not have any characteristic profiles, while only the $\mathrm{Na} / \mathrm{K}$ centric persistence diagram (Fig. 6b) has two profiles at $d_{k}$ $\sim 5$ and $\sim 10 \AA^{2}$. These profiles demonstrate that $\mathrm{Na}$ and $\mathrm{K}$ are highly correlated with edge-sharing oxygen in $\mathrm{Na} 50 \mathrm{~K} 50$ glass, similar to $\mathrm{Na}-\mathrm{O}-\mathrm{K}$. Indeed, the connectivity analysis ${ }^{46}$ confirmed that $81 \%$ of $\mathrm{Na}, \mathrm{K}$, and $\mathrm{O}$ atoms form a network in Na50K50. We compare the profiles shown in Fig. $6 \mathrm{~d}-\mathrm{f}$ with the partial pairdistribution functions, $g_{i j}(r)$, for the series of alkali silicate glasses in Fig. 6g. As seen in Fig. 6g (left), we can hardly observe differences among $g_{\mathrm{NaNa}}(r), g_{\mathrm{NaK}}(r)$, and $g_{\mathrm{KK}}(r)$ because they contain only 1-dimensional information. On the other hand, the three-body correlation as a function of distance in Fig. $6 \mathrm{~g}$ (right) shows the formation of specific correlations between $\mathrm{Na}$ and $\mathrm{K}$ ions, as mentioned above. Thus, the persistence diagram enables us to extract the characteristic topology in the glasses. To obtain more detailed structural information associated with the breakdown of the silicate network, alkali-oxygen polyhedra with the formation of edge-sharing NBO atoms in alkali silicate glasses are visualized in Fig. 7a-c. We believe that the visualization should provide us with crucial information to uncover the origin of the mixed alkali effect, as demonstrated in Fig. S1. Contrary to the similar schematic drawings in the previous studies ${ }^{21,25,26}$, we depicted the atomistic configurations from the structural models, which are consistent with both the NMR and the diffraction data, for the first time, provide us with more accurate information to reconcile the mixed alkali effect. A comparison between the Na100 and K100 glasses suggests that the shape of the Na-O polyhedra is planar, whereas that of the $\mathrm{K}-\mathrm{O}$ polyhedra is polyhedral. To understand the nature of the alkali-oxygen polyhedra, we calculated the coordination numbers of the bridging oxygen (BO) and NBO around the alkali ions, as summarized in Table 1 . The alkali-NBO coordination number is $\sim 2$ in both the Na100 and $\mathrm{K} 100$ glasses, and the alkali-BO coordination number is 2.1 in the Na100 glass. On the other hand, the K100 glass exhibits a larger coordination number, 2.4, and this trend is enhanced in the Na50K50 glass, in which the K$\mathrm{BO}$ coordination number is almost 3 . The $\mathrm{K}-\mathrm{O}$ polyhedra that are highly coordinated to the $\mathrm{BO}$ and $\mathrm{Na}-\mathrm{O}$ polyhedra are highlighted in black in Fig. $7 \mathrm{~b}$, where the potassium atom is trapped by a higher number of $\mathrm{BO}$ atoms (see Fig. $7 \mathrm{~d}$ ). It is likely that these specific configurations involving NBO atoms are a bottleneck in ionic conduction because the trapped potassium ions disturb ionic migration. It has been reported that each kind of alkali ion has an independent conduction pathway according to MD 

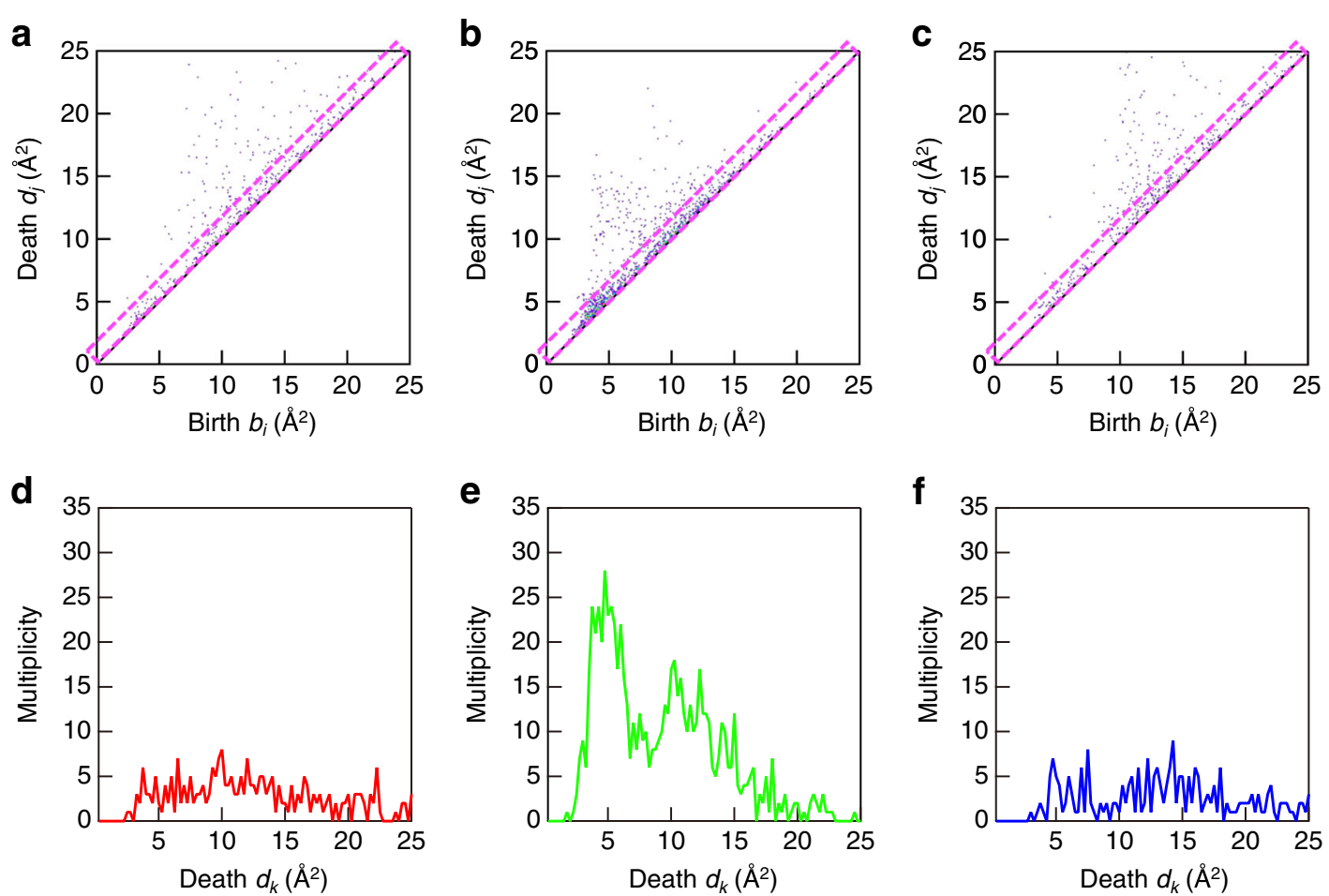

g
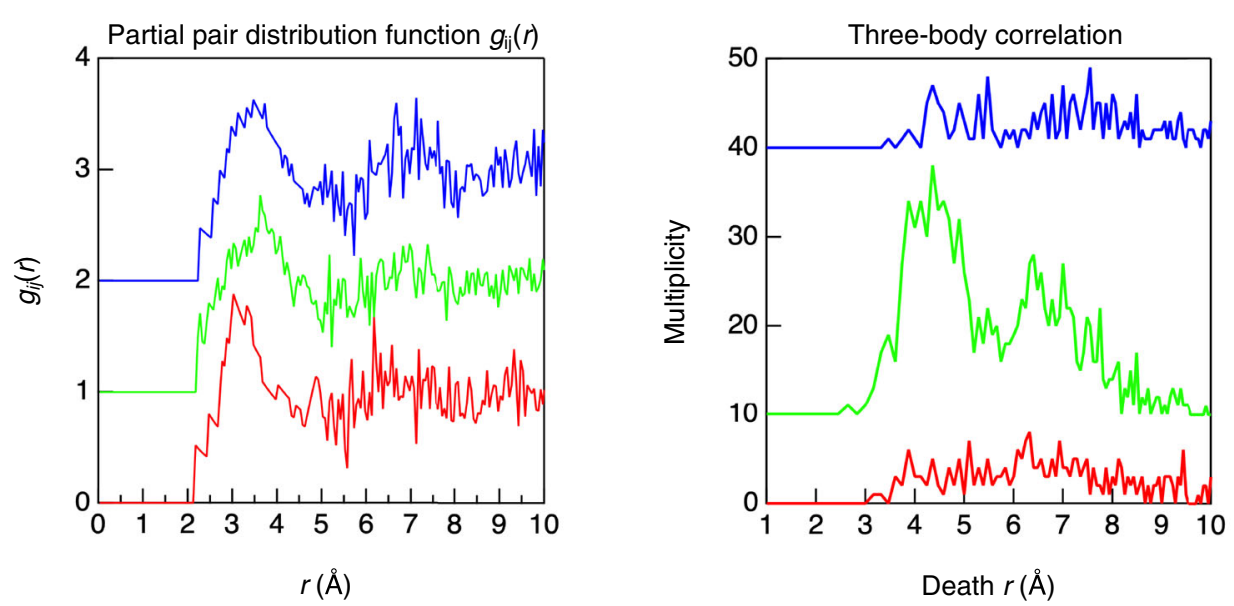

Fig. 6 The alkali-centric persistence diagrams for the Na50K50 glass. a Na-centric, b Na/K-centric, and $\mathbf{c}$ K-centric. The boxed regions follow the diagonal, and the associated profiles are plotted for $\mathbf{d} \mathrm{Na}$-centric, e $\mathrm{Na} / \mathrm{K}$-centric, and $\mathbf{f}$ K-centric. $\mathbf{g}$ Comparison between partial pair distribution functions, $g_{i j}(r)$ and three-body correlations extracted from the persistent diagram along with the diagonal lines for $\mathrm{Na}$-centric (red), Na/K-centric (green) and K-centric (blue).

simulations on lithium potassium metasilicate glass ${ }^{47}$. Accordingly, Habasaki et al. found that the transfer of alkali ions through different ion sites has a low probability and proposed an interpretation for restricted ion jumping among the sites occupied by other types of ions due to a mismatch in the occupation energies of unlike alkali ions ${ }^{48}$. Our intriguing finding; that is, the local environment of potassium ions in the highly coordinated $\mathrm{K}-\mathrm{O}$ polyhedra is significantly different from that of sodium ions in the four-coordinated $\mathrm{Na}-\mathrm{O}$ polyhedra, successfully proves their hypothesis. In addition, the specific correlations between the different ions, which are found in the Na50K50 glass by the persistent homology (Fig. 6g (right)) and connectivity analyses, profoundly inhibited the ionic migration in the Na50K50 glass further. Hence, the local structure associated with alkali connectivity is the intrinsic origin of the mixed alkali effect. In summary, we have succeeded in visualizing the modified network model proposed by Greaves with the bottleneck structure, which stands for the mixed alkali effect ${ }^{21}$. 


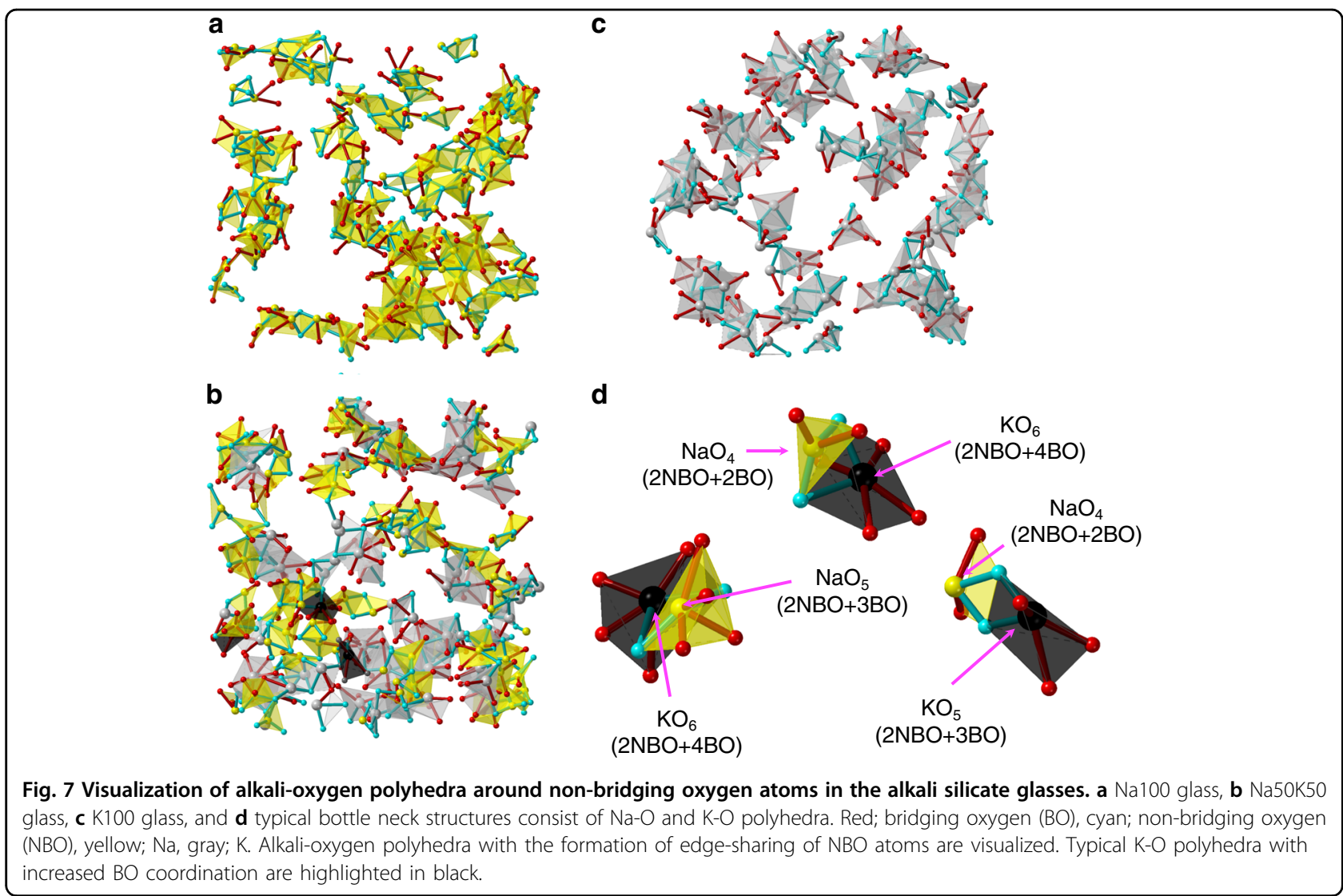

In this study, for the first time, we reconstructed the most reliable and accurate structure models for the alkali silicate glasses by using the combined RMC-MD method and reproducing the NMR spectra and the neutron and $\mathrm{X}$-ray structure factors simultaneously. On the basis of the most reliable structural model, we have succeeded in reconciling the mixed alkali effect based on the behaviors of the alkali ions around the $\mathrm{NBO}$ atoms, as well as the modification of the $\mathrm{Si}-\mathrm{O}$ network by addition of alkali oxides. The advanced topological analysis allows us to extract novel information on the structure to understand the origin of the mixed alkali effect. This dedicated approach therefore offers a way to find crucial knowledge to uncover other properties in glasses.

\footnotetext{
Acknowledgements

This work was partially supported by JST-PRESTO 'Advanced Materials Informatics through Comprehensive Integration among Theoretical, Experimental, Computational and Data-Centric Sciences', Japan Grant Number JPMJPR15N4 (to S.K.); the 'Materials Research by Information Integration' Initiative (MI ${ }^{2}$ ) project of the Support Program for Starting Up Innovation Hub from JST (To Y.O., S.K., and Y. H.); JST CREST 15656429 (to Y.H.); and JSPS KAKENHI Grant Number JP19K05648 (to Y.O.). The synchrotron radiation experiments were performed at BL04B2 of SPring-8 with the approval of the Japan Synchrotron Radiation Research Institute (JASRl; Proposal Nos. 2016A1499 and 2017B0134). The neutron experiment at the Materials and Life Science Experimental Facility of the J-PARC was performed under a user program (Proposal No. 2017A0146). We acknowledge Dr. Toshiya Otomo and Dr. Kazutaka Ikeda for their help in the neutron diffraction
}

measurement using the spectrometer NOVA at J-PARC. Dr. Hirokazu Masai is gratefully appreciated for helpful discussions.

\section{Author details}

${ }^{1}$ Center for Materials Research by Information Integration (CMI2), Research and Services Division of Materials Data and Integrated System (MaDIS) National Institute for Materials Science (NIMS), Ibaraki 305-0047, Japan. ${ }^{2}$ Institute for Integrated Radiation and Nuclear Science, Kyoto University,

2-1010 Asashiro-nishi, Kumatori-cho, Sennan-gun, Osaka 590-0494, Japan.

${ }^{3}$ Innovative Technology Laboratories, AGC Inc, 1150 Hazawa-cho, Yokohama 221-8755, Japan. ${ }^{4}$ Materials Integration Laboratories, AGC Inc, 1150 Hazawacho, Yokohama 221-8755, Japan. ${ }^{5}$ Center for Advanced Intelligence Project, RIKEN, 1-4-1 Nihonbashi, Chuo-ku, Tokyo 103-0027, Japan. ${ }^{6}$ CREST, Japan Science and Technology Agency, 7 Gobancho, Chiyoda-ku, Tokyo 102-0076, Japan. ${ }^{7}$ Kyoto University Institute for Advanced Study, WPI-ASHBi, Kyoto University, Yoshida Ushinomiya- cho, Sakyo-ku, Kyoto 606-8501, Japan. ${ }^{8}$ Research Center for Advanced Measurement and Characterization, NIMS, 1-11 Kouto, Sayo-cho, Sayo-gun, Hyogo 679-5148, Japan. ${ }^{9}$ PRESTO, Japan Science and Technology Agency, 7 Gobancho, Chiyoda-ku, Tokyo 102-0076, Japan. ${ }^{10}$ Diffraction and Scattering Division, Japan Synchrotron Radiation Research Institute (JASRI, SPring-8), 1-1-1 Kouto, Sayo-cho, Sayo-gun, Hyogo 679-5198, Japan

\section{Authors' contributions}

S.K. and YT. designed this research. Samples were prepared by H.H. and S.I. Dielectric constants were measured by H.H. Neutron diffraction measurements were performed by Y.T. and Y.O. High-energy X-ray diffraction measurements were conducted by Y.T., S.K, and Y.O. Molecular dynamics simulations were performed by T.T. and S.U. The obtained data were analyzed by S.K, Y.T., Y.O., S.F., I.O., and Y.H.

\section{Data availability}

The datasets generated during and/or analyzed during the current study are available from the corresponding author upon reasonable request. 


\section{Conflict of interest}

The authors declare that they have no conflict of interest.

\section{Publisher's note}

Springer Nature remains neutral with regard to jurisdictional claims in published maps and institutional affiliations.

Supplementary information is available for this paper at https://doi.org/ 10.1038/s41427-019-0180-4.

Received: 10 July 2019 Revised: 30 August 2019 Accepted: 19 September 2019.

Published online: 6 December 2019

\section{References}

1. Zachariasen, W. H. The atomic arrangement in glass. J. Am. Chem. Soc. 54, 3841-3851 (1932).

2. Elliott, S. R. The origin of the first sharp diffraction peak in the structure factor of covalent glasses and liquids. J. Phys. Condens. Matter 4, 7661-7678 (1992).

3. Salmon, P. S. Real space manifestation of the first sharp diffraction peak in the structure factor of liquid and glassy materials. Proc. R. Soc. Lond. A $\mathbf{4 4 5}$ 351-365 (1994).

4. Gaskell, P. H. \& Wallis, D. J. Medium-range order in silica, the canonical network glass. Phys. Rev. Lett. 75, 66-69 (1996).

5. Wright, A. C. Neutron scattering from vitreous silica. V. The structure of vitreous silica: What have we learned from 60 years of diffraction studies? J. Non-Cryst. Solids 179, 84-115 (1994).

6. Brüning, R. \& Cottrell, D. X-ray and neutron scattering observations of structural relaxation of vitreous silica. J. Non-Cryst. Solids 325, 6-15 (2003).

7. Susman, S. et al. Intermediate-range order in permanently densified vitreous $\mathrm{SiO}_{2}$ : a neutron-diffraction and molecular-dynamics study. Phys. Rev. B 43, 1194-1197 (1991).

8. Maekawa, H., Maekawa, T., Kawamura, K. \& Yokokawa, T. The structural groups of alkali silicate glasses determined from ${ }^{29}$ Si MAS-NMR. J. Non-Cryst. Solids 127, 53-64 (1991)

9. Du, J. \& Corrales, L. R. First sharp diffraction peak in silicate glasses: Structure and scattering length dependence. Phys. Rev. B 72, 092201 (2005).

10. Du, J. \& Corrales, L. R. Compositional dependence of the first sharp diffraction peaks in alkali silicate glasses: a molecular dynamics study. J. Non-Cryst. Solids 352, 3255-3269 (2006).

11. Wicks, J. D., McGreevy, R. L. \& Börjesson, L. A network problem: modelling alkali-silicate glasses with RMC. Phase Transit. 61, 195-213 (1997).

12. Hannon, A. C., Vessal, B. \& Parker, J. M. The structure of alkali silicate glasses. J. Non-Cryst. Solids 150, 97-102 (1992).

13. Florian, P., Vermillion, K. E., Grandinetti, P. J., Farnan, I. \& Stebbins, J. F. Cation distribution in mixed alkali disilicate glasses. J. Am. Chem. Soc. 118, 3493-3497 (1996).

14. Tokuda, Y., Oka, T., Takahashi, M. \& Yoko, T. Inhomogeneous distribution of $\mathrm{Na}^{+}$in alkali silicate glasses. J. Ceram. Soc. Jpn. 119, 909-915 (2011).

15. McMillan, P. Structural studies of silicate glasses and melts-applications and limitations of Raman spectroscopy. Am. Mineral. 69, 622-644 (1984).

16. Mysen, B. Physics and chemistry of silicate glasses and melts. Eur. J. Mineral. $\mathbf{1 5}$ 781-802 (2003).

17. Meyer, A., Horbach, J., Kob, W., Kargl, F. \& Schober, H. Channel formation and intermediate range order in sodium silicate melts and glasses. Phys. Rev. Lett. 93, 027801 (2004)

18. Kargl, F., Meyer, A., Koza, M. M. \& Schober, H. Formation of channels for fast-ion diffusion in alkali silicate melts: a quasielastic neutron scattering study. Phys. Rev. B 74, 014304 (2006)

19. Inoue, K., Kataoka, H., Nagai, Y., Hasegawa, M. \& Kobayashi, Y. Short and medium range order in two-component silica glasses by positron annihilation spectroscopy. J. Appl. Phys. 115, 204903 (2014).

20. Isard, J. O. The mixed alkali effects in glass. J. Non-Cnyst. Solids 1, 235-261 (1969).
21. Greaves, G. N. EXAFS and the structure of glass. J. Non-Cryst. Solids 71, 203-217 (1985).

22. Dyre, J. C., Maass, P., Roling, B. \& Sidebottom, D. L. Fundamental questions relating to ion conduction in disordered solids. Rep. Prog. Phys. 72, 046501 (2009).

23. Huang, C. \& Cormack, A. N. Structure and energetics in mixed-alkali-metal silicate glasses from molecular dynamics. J. Mater. Chem. 2, 281-287 (1992).

24. Swenson, J. \& Adams, S. Mixed alkali effect in glasses. Phys. Rev. B 47, 155507 (2003).

25. Greaves, G. N. \& Ngai, K. L. Reconciling ionic-transport properties with atomic structure in oxide glasses. Phys. Rev. B 52, 6358-6380 (1995).

26. Park, B. \& Cormack, A. N. Molecular dynamics simulations of structural changes in mixed alkali (Li $\mathrm{K} K$ ) silicate glasses. J. Non-Cnyst. Solids 255, 112-121 (1999).

27. Habasaki, J., Leon, C. \& Ngai, K. L. Dynamics of glassy, crystalline and liquid ionic conductors Springer International Publishing, Switzerland, 2017.

28. Otomo, T. et al. Fundamental research of hydrogen storage mechanism with high-intensity total diffractometer. KENS Rep. 17, 28-36 (2011).

29. Kohara, S. et al. Structural studies of disordered materials using high-energy $x$ ray diffraction from ambient to extreme conditions. J. Phys. Condens. Matter 19, 506101 (2007)

30. Faber, T. E. \& Ziman, J. M. A theory of the electrical properties of liquid metals III. The resistivity of binary alloys. Philos. Mag. 11, 153-173 (1965).

31. Lorch, E. Neutron diffraction by germania, silica and radiation-damaged silica glasses. J. Phys. C: Solid State Phys. 2, 229-237 (1969).

32. Gereben, O, Jóvári, P. Temleitner, L. \& Pusztai, L. A new version of the RMC+ + , reverse Monte Carlo programme, aimed at investigating the structure of covalent glasses. J. Optoelectron. Adv. Mater. 9, 3021-3027 (2007).

33. Evrard, G. \& Pusztai, L. Reverse Monte Calro modelling of the structure of disordered materials with RMC++: a new implementation of the algorithm in C. J. Phys. Condens. Matter 17, S1-S13 (2005).

34. Plimpton, S. Fast parallel algorithms for short-range molecular dynamics. J. Comput. Phys. 117, 1-19 (1995).

35. Pedone, A., Malavasi, G., Menziani, M. C., Cormack, A. N. \& Segre, U. A new selfconsistent empirical interatomic potential model for oxides, silicates, and silicabased glasses. J. Phys. Chem. B 110, 11780-11795 (2006).

36. Nosé, S. A unified formulation of the constant temperature molecular dynamics methods. J. Chem. Phys. 81, 511-519 (1984).

37. Roux, S. Le \& Jund, P. Ring statistics analysis of topological networks: New approach and application to amorphous $\mathrm{GeS}_{2}$ and $\mathrm{SiO}_{2}$ systems. Comput. Mater. Sci. 49, 70-83 (2010).

38. Heimbach et al. pyMolDyn: Identification, structure, and properties of cavities/ vacancies in condensed matter and molecules. J. Comput. Chem. 38, 389-394 (2017).

39. https:/www.wpi-aimr.tohoku.ac.jp/hiraoka_labo/homcloud/index.en.html.

40. Hiraoka, Y. et al. Hierarchical structures of amorphous solids characterized by persistent homology. Proc. Natl Acad. Sci. USA 113, 7035-7040 (2016).

41. Tomozawa, M. \& McGahay, V. Mechanism of the mixed alkali effect based upon the thermodynamic state of glass. J. Non-Cryst. Solids $\mathbf{1 2 8 , 4 8 - 5 6}$ (1991).

42. Salmon, P. S., Martin, R. A.r Mason, P. E. \& Cuello, G. J. Topological versus chemical ordering in network glasses at intermediate and extended length scales. Nature 435, 75-78 (2005)

43. Price, D. L. et al. Short-, intermediate-, and extended-range order in rubidium germanate glasses. Phys. Rev. B 55, 11249-11254 (1997).

44. Kohara, S. et al. Relationship between topological order and glass forming ability in densely packed enstatite and forsterite composition glasses. Proc. Natl Acad. Sci. USA 108, 14780-14785 (2011).

45. Gupta, P. K. \& Cooper, A. R. Topologically disordered networks of rigid polytopes. J. Non-Cryst. Solids 123, 14-21 (1990).

46. Kohara, S. et al. Lead silicate glasses: Binary network-former glasses with large amounts of free volume. Phys. Rev. B 82, 134209 (2010).

47. Habasaki, J., Okada, I. \& Hiwatari, Y. MD study of the mixed alkali effect in a lithium-potassium metasilicate glass. J. Non-Cryst. Solids 183, 12-21 (1995).

48. Habasaki, J., Okada, I. \& Hiwatari, Y. MD study of the mixed alkali effect in terms of the potential surface in the lithium-potassium metasilicate glass. J. NonCryst. Solids 208, 181-190 (1996). 